\title{
Long Time Behavior of Solutions to the Caginalp System with Singular Potential
}

\author{
Maurizio Grasselli, Hana Petzeltová and Giulio Schimperna
}

\begin{abstract}
We consider a nonlinear parabolic system which governs the evolution of the (relative) temperature $\vartheta$ and of an order parameter $\chi$. This system describes phase transition phenomena like, e.g., melting-solidification processes. The equation ruling $\chi$ is characterized by a singular potential $W$ which forces $\chi$ to take values in the interval $[-1,1]$. We provide reasonable conditions on $W$ which ensure that, from a certain time on, $\chi$ stays uniformly away from the pure phases 1 and -1 . Combining this separation property with the Łojasiewicz-Simon inequality, we show that any smooth and bounded trajectory uniformly converges to a stationary state and we give an estimate of the decay rate.
\end{abstract}

Keywords. Phase-field models, maximal monotone operators, comparison principle, asymptotic behavior, Łojasiewicz-Simon inequality

Mathematics Subject Classification (2000). Primary 35B40, secondary 34C11, $35 \mathrm{~K} 60,80 \mathrm{~A} 22$

\section{Introduction}

Let us consider a material occupying, for any time $t \geq 0$, a bounded and connected domain $\Omega \subset \mathbb{R}^{3}$ with a smooth boundary $\Gamma$. We suppose that the material under consideration has two phases like, for instance, solid and liquid or two different magnetization states, which are subject to temperature variations. Then, we denote by $\vartheta$ its (relative) temperature and by $\chi$ the order parameter (or phase-field) describing the proportion of either of the phases. A well-known system modelling the evolution of $\vartheta$ and $\chi$ has been proposed by Caginalp [14] for melting-solidification processes in several classes of materials. Taking all the

M. Grasselli: Dipartimento di Matematica, Politecnico di Milano, Via Bonardi, 9, I-20133 Milano, Italy; maugra@mate.polimi.it

H. Petzeltová: Mathematical Institute AS CR, Žitná, 25, CZ-115 67 Praha 1, Czech Republic; petzelt@math.cas.cz

Giulio Schimperna: Dipartimento di Matematica, Università di Pavia, Via Ferrata, 1, I-27100 Pavia, Italy; giusch04@unipv.it 
constants equal to 1 for the sake of simplicity, the resulting differential system has the following form:

$$
\begin{aligned}
\vartheta_{t}+\lambda(\chi)_{t}-\Delta \vartheta & =f \\
\chi_{t}-\Delta \chi+W^{\prime}(\chi) & =\lambda^{\prime}(\chi) \vartheta .
\end{aligned}
$$

We recall that $\vartheta$ is such that $\vartheta=0$ is the critical value at which the phase transition takes place. Regarding $\chi$, we observe that $\chi= \pm 1$ represent the pure states, while the values inside the open interval $(-1,1)$ account for local presence of a mixture (mushy region). Moreover, the function $\lambda(\cdot)$ stands for a latent heat density, $f$ is a volumic heat source and $W$ is the potential associated with the phase configuration.

In [14], $\lambda$ is assumed to be linear and $W$ is supposed to be a double well potential (i.e., $\left.W(y)=\left(y^{2}-1\right)^{2}\right)$. Initial and boundary problems for (1.1)-(1.2) with such a $W$ have been widely analyzed in the existing literature (see, e.g., $[13,39]$, cf. also $[9,11,12,18,24,34,35,36])$. Some papers have also been devoted to the treatment of more general potentials (see, for instance, [17, 45] for well-posedness results). Here we want to give a further contribution along this direction by examining the longtime behavior of solutions when the potential $W$ has a domain contained in $[-1,1]$. This means that the values outside $[-1,1]$, which are not physically meaningful, are excluded by setting $W(y):=+\infty$ for $y \notin[-1,1]$ so justifying the adjective singular in the title. Our potential $W$ is the sum of a principal part $\widehat{\beta}$, assumed to be convex and accounting for the presence of the barriers at \pm 1 , and of a possibly nonconvex but smooth remainder $\widehat{\gamma}$. In this regard, the derivative $W^{\prime}$ in (1.2) has in fact to be intended as a subdifferential $\partial W$ in the sense of Convex Analysis (see, e.g., $[8,10]$ ) and relation (1.2) has to be understood as a differential inclusion due to the possibly multivalued character of $\partial W=\partial \widehat{\beta}+\widehat{\gamma}^{\prime}$. In this general setting, a physically relevant case is, e.g., the double obstacle potential; namely the convex part $\widehat{\beta}$ is the indicator function of $[-1,1]$, which is defined as identically 0 inside that interval, and $+\infty$ outside. In such a situation, although $W$ is very regular in the open interval $(-1,1)$, since it coincides with its smooth part $\widehat{\gamma}$, we cannot in general exclude that, at some point $(x, t)$, the variable $\chi(x, t)$ might take either of the values \pm 1 corresponding to the barriers. As we shall see below, it would be interesting to know sufficient conditions on $W$ which ensure that $\chi$ stays (uniformly) away from \pm 1 from a certain time on. This is exactly the main goal of the present paper. To be more precise, we first endow system (1.1)-(1.2) with the physically reasonable boundary conditions

$$
\begin{aligned}
\vartheta=0 & \text { on } \Gamma \times(0,+\infty) \\
\chi_{\boldsymbol{n}}=0 & \text { on } \Gamma \times(0,+\infty),
\end{aligned}
$$


$\boldsymbol{n}$ being the outward normal to $\Gamma$, and the initial conditions

$$
\begin{array}{ll}
\vartheta(0)=\vartheta_{0} & \text { in } \Omega \\
\chi(0)=\chi_{0} & \text { in } \Omega .
\end{array}
$$

Our main result will be to find a wide class of data and potentials for which the following separation property holds:

$$
\begin{aligned}
& \text { there exist } T>0 \text { and } \delta \in(0,1) \text { such that } \\
& -1+\delta<\chi(x, t)<1-\delta \text { for all } x \in \Omega, t \geq T \text {, }
\end{aligned}
$$

where $(\vartheta, \chi)$ is the unique global solution to problem $(1.1)-(1.6)$. It is worth mentioning that the importance of this property has already been analyzed in [40] for the Cahn-Hilliard equation.

In order to understand for which class of potentials we can hope to obtain $(\mathrm{S})$, we introduce the energy functional

$$
E(u, v):=\int_{\Omega}\left(\frac{(u-\lambda(v))^{2}}{2}+\frac{|\nabla v|^{2}}{2}+W(v)\right) \mathrm{d} x
$$

which can be interpreted as a real valued functional, defined on the space $\mathcal{E}:=H^{-1}(\Omega) \times L^{2}(\Omega)$, provided that the integral is intended to be $+\infty$ when either $u$ is not in $L^{2}(\Omega)$, or $v$ is not in $H^{1}(\Omega)$, or $W(u)$ is not summable. We notice anyway that $E$ is bounded from below, and can actually be assumed nonnegative, thanks to the convex character of the principal part of $W$.

It is now not difficult to verify that, introducing the auxiliary variable $e:=$ $\vartheta+\lambda(\chi)$ (i.e., the enthalpy of the system), and setting for the moment $f \equiv 0$, our boundary value problem can be restated in the gradient flow form

$$
(e, \chi)_{t}=-\partial E(e, \chi) \quad \text { for } t \geq 0
$$

the symbol $\partial$ denoting here the subdifferential in the space $\mathcal{E}$. Owing to the coercivity of $E$ and to the compactness of its sublevels with respect to the $\mathcal{E}$ norm, it is then apparent that a solution exists at least for initial data $\left(e_{0}, \chi_{0}\right)$, $e_{0}=\vartheta_{0}+\lambda\left(\chi_{0}\right)$, of bounded energy, i.e., such that $E\left(e_{0}, \chi_{0}\right)<+\infty$; moreover, in absence of an external source, $E(t)=E(e(t), \chi(t))$ tends to decrease along solution paths. Consequently, if at least one of the pure configurations corresponding to \pm 1 is a local minimum of the energy, there is no hope that the system may tend to move away from it, i.e., to get the separation property. Conversely, if the leftmost and the rightmost minima of $W$ are well separated from -1 and +1 , respectively (cf. condition ( $\mathrm{H} 7 \mathrm{w}$ ) below for a rigorous statement of this fact), then the variable $\chi$, as it approaches these minima, moves away from the values \pm 1 , a clear indication that $(S)$ might be valid. 
In this paper we shall show that, if $W$ is sufficiently coercive at the extrema (corresponding, roughly speaking, to the presence of "deep" minima inside $(-1,1)$ ), in a way compatible with the other data (in particular, with the source $f$ which has to be not too big as $t$ grows), then the solution moves away from \pm 1 in the $L^{\infty}(\Omega)$-norm. This implies (S) at least for a sufficiently large critical time $T$. We point out that the compatibility condition required to get $(\mathrm{S})$ does not involve, instead, the initial data, which can have arbitrarily large energy and influence the critical time $T$ and $\delta$ only.

The proof of this result is achieved by means of a very simple argument. Firstly, we prove uniform parabolic regularization estimates. At this step, we have to take care of the fact that (S) is not yet known, and it might well happen that, for instance, $\chi(t) \equiv 1$ in some region of nonzero spatial measure and for some time $t>0$. Thus, the estimates have to respect the upper regularity threshold imposed by the presence of the barriers. However, the regularizing effect is sufficiently strong to get, starting from any (arbitrarily small) time $r>0$, a uniform (in time) bound of the $L^{\infty}(\Omega)$-norm of both $\vartheta$ and $\chi$. This allows us to compare our $\chi$, for $t \geq r$, with the solutions of the ODEs obtained by suppressing the Laplace operator in (2.13) and replacing the right hand side with (plus and minus) its $L^{\infty}(\Omega)$-norm, which is now a uniformly bounded quantity. Now, in order that the solution to each ODE has the right profile, entailing validity of $(\mathrm{S})$, we need $\lambda^{\prime}(\chi) \vartheta$ be small enough in $L^{\infty}(\Omega)$, in relation to the coercivity property of $W$, i.e., the above mentioned compatibility condition.

We have to remark that, when $W$ is "very coercive" at the extrema, i.e., the leftmost and rightmost minima are "infinitely deep" (namely, $W$ is $+\infty$ outside the interval $(-1,1)$ ), then no compatibility condition is needed, and the parabolic regularization property is sufficiently strong to guarantee the validity of $(\mathrm{S})$ for any choice of the critical time $T>0$. Also, no compatibility is needed when the heat source $f$ is $L^{2}$ in time. Here the comparison argument works under very general assumptions on $W$; however, in this case $T$ is not arbitrary and there might be a transient dynamics where $\chi$ is not completely separated from the pure states. If the heat source vanishes after a certain given time, the property (S) allows us to prove a further result of this note, namely, the convergence of each bounded solution of (1.1)-(1.6) to a stationary state. Indeed, it is well known (and actually not difficult to prove) that, as $\left(\vartheta_{0}, \chi_{0}\right)$ have finite energy, then there exists the $\omega$-limit of the system, all of whose elements have the form $\left(0, \chi_{\infty}\right), \chi_{\infty}$ being one solution of the homogeneous Neumann problem for the nonlinear elliptic equation (more precisely, inclusion)

$$
-\Delta v+\partial W(v) \ni 0 .
$$

However, if $W$ is not convex, as it happens in our case, it is known that such a problem may have a continuum of solutions (see, e.g., [26, Remark 2.3.13]). 
Consequently, it is a nontrivial question to understand if the $\omega$-limit is a singleton $\chi_{\infty}$, i.e., if all the trajectory $\chi(t)$ converges to this single stationary state as $t \nearrow \infty$. This property has been proved in [2] for the Caginalp system (1.1)-(1.2) with double well potential, subject to homogeneous Dirichlet boundary conditions, provided that $W$, whose domain is now the whole real line, is analytic on a sufficiently large interval. More recently, in [49] a similar result has been obtained in the more difficult case of homogeneous Neumann boundary conditions. The method used in $[2,49]$ essentially relies on a sharp growth estimate, originally established by Łojasiewicz $[37,38]$ for analytic functions of several complex variables. It has been then shown by Simon [46] (see also [32]) that this inequality can be extended to an infinite dimensional setting and used to characterize the $\omega$-limit sets of various types of evolution systems with analytic nonlinearities. However, it is worth reminding that if $W$ is just $C^{\infty}$, then the $\omega$-limit set might be a continuum (see [42], cf. also [7, 41]).

Several (nontrivial) variants of the Łojasiewicz-Simon method have been applied in these last years to many other systems of PDEs (see, e.g., [1, 3, 4, $5,15,16,19,22,20,27,28,31,33,44,48]$ ), among which phase-field models are just meaningful examples. It is also worth mentioning that a non-smooth version of this inequality has been obtained in [21] and applied to a spatially nonlocal phase-field system. From this point of view, we have to remark that the singleton property for our Caginalp system with singular potential is just a consequence of the separation property $(\mathrm{S})$ combined with some results in [2], provided of course that the restriction of $W$ to the open interval $(-1,1)$ is analytic and $f(t)$ goes to zero in an appropriate way. Though we do not claim any mathematical originality for this step, we believe nonetheless that the property itself is interesting enough to be stated as a theorem. Moreover, we also give an estimate of the decay rate to equilibrium.

In the next Section 2 we shall state our hypotheses and we present the main results which will be proved in Section 3 .

\section{Main results}

Let us set $H:=L^{2}(\Omega)$ endowed with its standard scalar product $(\cdot, \cdot)$. The same notation will be also used for $\left(L^{2}(\Omega)\right)^{3}$. Then we introduce

$$
V_{0}:=H_{0}^{1}(\Omega), \quad V:=H^{1}(\Omega),
$$

and endow them with their standard scalar products, namely

$$
(u, v)_{V_{0}}:=(\nabla u, \nabla v), \quad(u, v)_{V}:=(\nabla u, \nabla v)+(u, v) .
$$

We recall that $V_{0} \hookrightarrow V \hookrightarrow H \equiv H^{\prime} \hookrightarrow V^{\prime} \hookrightarrow V_{0}^{\prime}$, where $V_{0}^{\prime}=H^{-1}(\Omega)$. Let us now consider the linear operators

$$
A: V \rightarrow V^{\prime}, \quad\langle A v, z\rangle:=(\nabla v, \nabla z) \quad \text { for all } z \in V
$$




$$
B: V_{0} \rightarrow V_{0}^{\prime}, \quad\langle B v, z\rangle:=(\nabla v, \nabla z) \quad \text { for all } z \in V_{0} .
$$

Note that $A$ is weakly elliptic, while $B$ is strongly elliptic thanks to the Poincaré inequality

$$
\|v\|_{H} \leq c_{\Omega}\|\nabla v\|_{H} \quad \text { for all } v \in V_{0}
$$

where $c_{\Omega}$ is a suitable positive constant only depending on $\Omega$. We also consider the domains of the $H$-realizations of the operators $A$ and $B$

$$
D(A)=\left\{v \in H^{2}(\Omega): v_{\boldsymbol{n}}=0 \text { on } \Gamma\right\}, \quad D(B)=H^{2}(\Omega) \cap V_{0},
$$

and we endow them with their graph norms.

Our basic hypotheses on the data are the following:

$$
\begin{aligned}
& \lambda \in C^{2}(\mathbb{R}), \quad \gamma \in C^{1}(\mathbb{R}) \\
& \vartheta_{0} \in H \\
& \beta \subset \mathbb{R} \times \mathbb{R} \text { is a maximal monotone graph such that } 0 \in \beta(0) \\
& \overline{\operatorname{dom}(\beta)}=[-1,1] .
\end{aligned}
$$

We let

$$
L:=\sup _{y \in[-1,1]}\left\{\gamma(y), \gamma^{\prime}(y), \lambda(y), \lambda^{\prime}(y), \lambda^{\prime \prime}(y)\right\} .
$$

Next, we denote by $\widehat{\gamma}$ a primitive of $\gamma$ that is nonnegative in $[-1,1]$, and by $\widehat{\beta}: \mathbb{R} \rightarrow[0,+\infty]$ the convex and lower semicontinuous function such that $\widehat{\beta}(0)=0$ and $\beta=\partial \widehat{\beta}$. Then, we set $W:=\widehat{\beta}+\widehat{\gamma}$, so that $\partial W=\beta+\gamma$. Thanks to (H4), $W$ is identically $+\infty$ outside $[-1,1]$ and it is never $+\infty$ inside $(-1,1)$. Thus, our assumptions (H3)-(H4) are just a reformulation of what we required to $W$ in the Introduction. Also, observe that, thanks to (H1) and (H3), there exists a constant $c_{\gamma}>0$ such that

$$
0 \leq \widehat{\gamma}(y) \leq c_{\gamma}, \quad 0 \leq W(y) \leq \widehat{\beta}(y)+c_{\gamma} \leq W(y)+c_{\gamma} \quad \forall y \in[-1,1],
$$

and

$$
z y \geq \widehat{\beta}(y) \quad \forall y \in[-1,1], \forall z \in \beta(y) .
$$

We can now state our assumptions on the initial datum $\chi_{0}$, namely,

$$
\chi_{0} \in V, \quad \widehat{\beta}\left(\chi_{0}\right) \in L^{1}(\Omega) .
$$

Of course, the second condition is equivalent to requiring $W\left(\chi_{0}\right) \in L^{1}(\Omega)$. It is apparent that the coupling of (H2) and (H5) is a restatement of the finite energy assumption $E\left(e_{0}, \chi_{0}\right)<+\infty$ mentioned in the Introduction.

Finally, let us detail our assumptions on the heat source. We introduce the Banach space of $L_{\text {loc }}^{2}$-translation bounded functions with values in a Banach space $X$ :

$$
\mathcal{T}^{2}(X):=\left\{g \in L_{\mathrm{loc}}^{2}([0,+\infty) ; X):\|g\|_{\mathcal{T}^{2}(X)}^{2}:=\sup _{t \geq 0} \int_{t}^{t+1}\|g(s)\|_{X}^{2} \mathrm{~d} s<+\infty\right\},
$$


and we suppose that

$$
f \in \mathcal{T}^{2}(V) .
$$

This assumption is satisfied when $f$ is time-independent (i.e., $f$ is a fixed element of $V$ ) and it is essential for getting uniform-in-time estimates, since it does not allow the $L^{\infty}(\Omega)$-norm of $\vartheta$ to get too large.

Let us now state our first theorem, which is devoted to the well-posedness of the weak formulation of problem (1.1)-(1.6) as well as to some parabolic regularization properties of its solution. The first part of the result is basically known; thus, the proof will be mainly concerned with the second part.

Theorem 2.1. Let (H1)-(H6) hold. Then there exists a unique triplet $(\vartheta, \chi, \xi)$ such that

$$
\begin{aligned}
& \vartheta \in H_{\mathrm{loc}}^{1}\left([0,+\infty) ; V_{0}^{\prime}\right) \cap C^{0}([0,+\infty) ; H) \cap L_{\mathrm{loc}}^{2}\left([0,+\infty) ; V_{0}\right) \\
& \chi \in H_{\mathrm{loc}}^{1}([0,+\infty) ; H) \cap C^{0}([0,+\infty) ; V) \cap L_{\mathrm{loc}}^{2}([0,+\infty) ; D(A)) \\
& \xi \in L_{\mathrm{loc}}^{2}([0,+\infty) ; H) \\
& -1 \leq \chi \leq 1 \quad \text { a.e. in } \Omega \times(0,+\infty), \\
& \widehat{\beta}(\chi) \in L^{\infty}\left(0,+\infty ; L^{1}(\Omega)\right),
\end{aligned}
$$

which satisfies, for almost all $t \in(0,+\infty)$,

$$
\begin{aligned}
\vartheta_{t}+\lambda(\chi)_{t}+B \vartheta & =f & & \text { in } V_{0}^{\prime} \\
\chi_{t}+A \chi+\xi+\gamma(\chi) & =\lambda^{\prime}(\chi) \vartheta & & \text { in } V^{\prime} \\
\xi & \in \beta(\chi) & & \text { a.e. in } \Omega,
\end{aligned}
$$

together with the initial conditions

$$
\vartheta(0)=\vartheta_{0}, \quad \chi(0)=\chi_{0}, \quad \text { a.e. in } \Omega .
$$

Moreover, for all $r \in(0,1]$, there exists a constant $K_{0}$ depending on $\vartheta_{0}, \chi_{0}, f$, $\gamma, \lambda$, on $r$, but neither on $\beta$ nor on $t$, such that, for any $t \geq 3 r$,

$$
\|\vartheta(t)\|_{C(\bar{\Omega})}+\|\vartheta(t)\|_{V_{0}}+\|\chi(t)\|_{D(A)}+\left\|\chi_{t}(t)\right\|_{H}+\left\|\beta^{0}(\chi(t))\right\|_{H} \leq K_{0} .
$$

Here by $\beta^{0}$ we denote the minimal section of $\beta$ (see, e.g., [10]).

Let us examine now the separation property. We have to refine our assumptions on the potential $W$. We set

$$
R^{ \pm}:= \begin{cases}\beta^{0}( \pm 1)+\gamma( \pm 1) & \text { if } \pm 1 \in \operatorname{dom}(\beta) \\ \pm \infty & \text { otherwise. }\end{cases}
$$

Then, our weak coercivity assumption on $W$ can be stated as

$$
R:=\min \left\{R^{+},-R^{-}\right\}>0 .
$$


Correspondingly, we say that $W$ is strongly coercive if

$$
R:=\min \left\{R^{+},-R^{-}\right\}=+\infty,
$$

which is of course equivalent to requiring that $\operatorname{dom}(\beta)=(-1,1)$.

Remark 2.2. A meaningful example of potential $W$ satisfying (H7s) is the so-called logarithmic potential, namely,

$$
W(y)=k_{1}((1+y) \ln (1+y)+(1-y) \ln (1-y))-\frac{k_{2}}{2} y^{2}+k_{3} y+k_{4},
$$

for $y \in(-1,1)$, where $k_{i} \in \mathbb{R}, i=1 \div 4$, with $k_{1}$ and $k_{2}$ positive. In this case, our model can be viewed as a linearized version (with respect to the absolute temperature around the critical one) of the Penrose-Fife system analyzed in [30] (see also [43]). There, a separation property is established on a finite time interval.

Our main result is

Theorem 2.3. Assume (H1)-(H6) and let $(\vartheta, \chi)$ be the solution to $(2.7)-(2.15)$. There exists $R_{0} \geq 0$, depending on $\Omega, \lambda, \gamma, f$ such that, if $(\mathrm{H} 7 \mathrm{w})$ holds with $R>R_{0}$, then the separation property $(\mathrm{S})$ takes place.

Remark 2.4. As it will appear clear in the proof, the separation time $T$ as well as the distance $\delta$ actually depend on $R, R_{0}$ and on the initial data.

Next, as mentioned in the Introduction, we have the following couple of consequences related to particular (but significant) cases.

Corollary 2.5. Let (H1)-(H6) and (H7s) hold. Then, the property $(\mathrm{S})$ holds for any time $T>0$ and for a suitable $\delta>0$ depending on $T$ and on all the other data.

Corollary 2.6. Let $(\mathrm{H} 1)-(\mathrm{H} 6)$ and $(\mathrm{H} 7 \mathrm{w})$ hold. If

$$
f \in L^{2}([0,+\infty) ; H),
$$

then Theorem 2.3 is valid with $R_{0}=0$.

Finally, we state the result concerning the long time behavior.

Theorem 2.7. Assume $(\mathrm{H} 1)-(\mathrm{H} 6)$ and $(\mathrm{H} 8)$. Then, as $t$ tends to $+\infty$, we have

$$
\vartheta(t) \rightarrow 0 \quad \text { strongly in } V \cap C(\bar{\Omega}),
$$

and the $\omega$-limit set of the $\chi$-trajectory is contained in the set of solutions to the equation

$$
A v+\partial W(v) \ni 0
$$


If $(\mathrm{H} 7 \mathrm{w})$ holds, the restriction of $W$ to $(-1,1)$ is real analytic, and $f \equiv 0$, then the $\omega$-limit set consists of a single point $\chi_{\infty}$, i.e., as $t$ goes to $+\infty$,

$$
\chi(t) \rightarrow \chi_{\infty} \quad \text { strongly in } V \cap C(\bar{\Omega}) .
$$

Moreover, there exist $\rho \in\left(0, \frac{1}{2}\right), t^{*}>0$, and a constant $c$ depending on $\rho, \lambda, \gamma, L$ and $\Omega$ such that, for any $t>t^{*}$,

$$
\|\vartheta(t)\|_{H}+\left\|\chi(t)-\chi_{\infty}\right\|_{H} \leq c(1+t)^{-\frac{\rho}{1-2 \rho}}
$$

Remark 2.8. Theorem 2.7 still holds when $f$ is asymptotically autonomous in the following sense (see $[16,31])$ :

$$
\sup _{t \geq 0} t^{1+\varpi} \int_{t}^{\infty}\|f(s)\|^{2} d s<\infty
$$

for some $\varpi>0$. One can argue, for instance, as in [25]. Of course, the decay rate will also depend on $\varpi$.

Remark 2.9. Let $f \equiv 0$ for the sake of simplicity. It is worth observing that the estimates obtained in the subsequent proof of Theorem 2.1 (see Section 3) along with the arguments used in [43, Section 4] allow us to construct a strongly continuous semigroup $S(t)$ on the phase space

$$
\mathcal{X}:=\left\{(u, v) \in H \times V: \hat{\beta}(v) \in L^{1}(\Omega)\right\},
$$

endowed with the complete metric

$$
d_{\mathcal{X}}\left(\left(u_{1}, v_{1}\right),\left(u_{2}, v_{2}\right)\right):=\left\|u_{1}-u_{2}\right\|_{H}+\left\|v_{1}-v_{2}\right\|_{V}+\int_{\Omega}\left|\hat{\beta}\left(v_{1}\right)-\hat{\beta}\left(v_{2}\right)\right|,
$$

such that $(\vartheta(t), \chi(t))=S(t)\left(\vartheta_{0}, \chi_{0}\right)$ solves $(2.12)-(2.15)$. Moreover, still arguing as in [43], we can prove that $S(t)$ is dissipative (i.e., it admits a bounded absorbing set in $\mathcal{X}$ ) and it possesses a (compact and connected) global attractor $\mathcal{A}$ which is bounded in $\mathcal{H}=V_{0} \times D(A)$. Moreover, since the system has a global Lyapunov functional (see below (3.1)), then it is well known that

$$
\mathcal{A}=\left\{\left(\vartheta_{0}, \chi_{0}\right) \in \mathcal{H}: \begin{array}{l}
\text { the trajectory } \mathbf{z}(t) \text { originated from }\left(\vartheta_{0}, \chi_{0}\right) \text { is } \\
\text { in } \mathcal{X}, \text { for some } \chi_{\infty} \text { satisfying }(2.18) .
\end{array}\right\}
$$

Thus, if $W$ satisfies the assumptions of Theorem 2.7, we can conclude that $\mathcal{A}$ consists of all the pairs $\left(\vartheta_{0}, \chi_{0}\right) \in \mathcal{H}$ which originate either constant or heteroclinic orbits. 
Remark 2.10. As it will be clear from the proof, the singleton property (2.19) for the $\omega$-limit does not directly depend on Theorem 2.3, since the properties of the solutions to (2.18) (cf. (3.42) below) and the precompactness in $C(\bar{\Omega})$ of $\chi$-trajectories (which follows from (2.8) and (2.16)) are enough to get (S) at least from a sufficiently large $T$. Nevertheless, Theorem 2.3 permits to get a quantitative estimate of the time $T$ after which the trajectories start being separated and, consequently, the Łojasiewicz-Simon inequality can be applied. We also remark that, in the statement of Theorem 2.7, analyticity of $W$ might be required only in an open interval containing all the zeros of $W^{\prime}$. We supposed it holds in $(-1,1)$ just to avoid further technicalities in the proof.

\section{Proofs}

3.1. Proof of Theorem 2.1. If $\lambda$ is linear, existence and uniqueness follows from [45, Theorem 5.1]. On the other hand, the arguments used to get the quoted result can be extended to the case of a nonlinear $\lambda$ satisfying (H1) with no essential difficulties. Therefore, in the sequel we focus on the proof of property (2.16). For the sake of simplicity, we shall perform only formal a priori estimates. However, these can be justified rigorously by using, for instance, a suitable approximation scheme based on the Faedo-Galerkin method combined with a Yosida regularization of $\beta$.

First estimate. Let us test $(2.12)$ by $\vartheta,(2.13)$ by $\chi_{t}$, and take the sum. Noting that a couple of terms cancels out and using Young and Poincaré inequalities, we then get

$$
\frac{\mathrm{d}}{\mathrm{d} t}\left(\frac{\|\vartheta\|_{H}^{2}}{2}+\frac{\|\nabla \chi\|_{H}^{2}}{2}+\int_{\Omega} W(\chi) \mathrm{d} x\right)+\frac{\|\vartheta\|_{V_{0}}^{2}}{2}+\left\|\chi_{t}\right\|_{H}^{2} \leq \frac{c_{\Omega}^{2}}{2}\|f\|_{H}^{2} .
$$

Let us now test (2.13) by $\chi$. Using (H1), (2.5), (2.6), the uniform bound $-1 \leq \chi \leq 1$, and, again, Young and Poincaré inequalities, we obtain

$$
\frac{\mathrm{d}}{\mathrm{d} t} \frac{\|\chi\|_{H}^{2}}{2}+\|\nabla \chi\|_{H}^{2}+\int_{\Omega} W(\chi) \mathrm{d} x \leq \frac{\|\vartheta\|_{V_{0}}^{2}}{4}+c
$$

We point out that, here and below, the symbol $c$ will be used to denote a generic positive constant, which may vary from line to line (even within the same formula), which depends on $\lambda, \gamma, L, \Omega$ at most. The generic constant additionally depending on $\|f\|_{\mathcal{T}^{2}(V)}$ will be indicated by $c_{f}$.

Adding together (3.1) and (3.2), using once more the Poincaré inequality, and adding to both sides the $H$-norm of $\chi$ (which is uniformly bounded), we 
get

$$
\begin{aligned}
& \frac{\mathrm{d}}{\mathrm{d} t}\left(\frac{\|\vartheta\|_{H}^{2}}{2}+\frac{\|\nabla \chi\|_{H}^{2}}{2}+\int_{\Omega} W(\chi) \mathrm{d} x\right)+\frac{\|\vartheta\|_{V_{0}}^{2}}{8} \\
& +\frac{1}{8 c_{\Omega}^{2}}\|\vartheta\|_{H}^{2}+\left\|\chi_{t}\right\|_{H}^{2}+\|\chi\|_{V}^{2}+\int_{\Omega} W(\chi) \mathrm{d} x \leq c\left(1+\|f\|_{H}^{2}\right) .
\end{aligned}
$$

Denoting now (cf. (1.7))

$$
E(t):=\frac{\|\vartheta\|_{H}^{2}}{2}+\frac{\|\nabla \chi\|_{H}^{2}}{2}+\int_{\Omega} W(\chi) \mathrm{d} x,
$$

we can rewrite (3.3) as

$$
\frac{\mathrm{d}}{\mathrm{d} t} E(t)+\varepsilon E(t)+\frac{\|\vartheta(t)\|_{V_{0}}^{2}}{8}+\left\|\chi_{t}(t)\right\|_{H}^{2} \leq c\left(1+\|f(t)\|_{H}^{2}\right),
$$

where we have set $\varepsilon:=\min \left\{1,1 / 4 c_{\Omega}^{2}\right\}$. Hence, using the Gronwall Lemma in differential form (cf., e.g., [23, Lemma 2.5]), we deduce, for any $t>0$,

$$
\begin{aligned}
E(t) & \leq E(0) e^{-\varepsilon t}+c\left(\varepsilon^{-1}+\int_{0}^{t}\|f(s)\|_{V}^{2} e^{-\varepsilon(t-s)} \mathrm{d} s\right) \\
& \leq E(0) e^{-\varepsilon t}+c_{f} .
\end{aligned}
$$

Furthermore, integrating (3.5) between $t$ and $t+r$, where $t \geq 0$ and $r \in(0,1]$, and taking (3.6) into account, it is not difficult to infer

$$
\begin{aligned}
E(t+r)+\int_{t}^{t+r}\left(\frac{1}{8}\|\vartheta(s)\|_{V_{0}}^{2}\right. & \left.+\left\|\chi_{t}(s)\right\|_{H}^{2}\right) \mathrm{d} s \\
& \leq E(t)+c\left(r+\|f\|_{L^{2}(t, t+r ; H)}^{2}\right) \\
& \leq E(0) e^{-\varepsilon t}+c_{f} .
\end{aligned}
$$

Second estimate. Let us now test $(2.12)$ by $\vartheta_{t}$. We easily obtain

$$
\frac{\left\|\vartheta_{t}\right\|_{H}^{2}}{2}+\frac{\mathrm{d}}{\mathrm{d} t} \frac{\|\vartheta\|_{V_{0}}^{2}}{2} \leq \frac{1}{2} \int_{\Omega}\left(f-\lambda^{\prime}(\chi) \chi_{t}\right)^{2} \mathrm{~d} x
$$

so that, recalling (3.7), the uniform Gronwall Lemma (cf., e.g., [47, Lemma III.1.1]) yields

$$
\|\vartheta(t+r)\|_{V_{0}}^{2} \leq c\left(E(0) e^{-\varepsilon t}+c_{f}\right)\left(1+r^{-1}\right) \text { for all } t \geq 0
$$

Next, integrating $(3.8)$ over $(t, t+r)$, we also get

$$
\int_{t}^{t+r}\left\|\vartheta_{t}(s)\right\|_{H}^{2} \mathrm{~d} s \leq c\left(E(0) e^{-\varepsilon t}+c_{f}\right)\left(1+r^{-1}\right) \quad \text { for all } t \geq r .
$$


Third estimate. A formal differentiation with respect to time of (2.13) gives

$$
\chi_{t t}+A \chi_{t}+W^{\prime \prime}(\chi) \chi_{t}=\lambda^{\prime \prime}(\chi) \chi_{t} \vartheta+\lambda^{\prime}(\chi) \vartheta_{t}
$$

where, of course, the second derivative $W^{\prime \prime}$ does not make sense in the present setting, but still the procedure can be made rigorous by regularizing $\beta$. Now, let us test (3.11) by $\chi_{t}$. Thanks to the monotonicity of $\beta$, we have

$$
\frac{\mathrm{d}}{\mathrm{d} t} \frac{\left\|\chi_{t}\right\|_{H}^{2}}{2}+\left\|\nabla \chi_{t}\right\|_{H}^{2} \leq \int_{\Omega}\left(-\gamma^{\prime}(\chi) \chi_{t}^{2}+\lambda^{\prime \prime}(\chi) \chi_{t}^{2} \vartheta+\lambda^{\prime}(\chi) \vartheta_{t} \chi_{t}\right) \mathrm{d} x .
$$

Using (H1), estimating the second term as follows,

$$
\int_{\Omega} \lambda^{\prime \prime}(\chi) \chi_{t}^{2} \vartheta \mathrm{d} x \leq \frac{\left\|\chi_{t}\right\|_{V}^{2}}{2}+c\|\vartheta\|_{V_{0}}^{2}\left\|\chi_{t}\right\|_{H}^{2},
$$

and adding $\frac{1}{2}\left\|\chi_{t}\right\|_{H}^{2}$ to both sides of (3.12), we easily deduce

$$
\frac{\mathrm{d}}{\mathrm{d} t}\left\|\chi_{t}\right\|_{H}^{2}+\left\|\chi_{t}\right\|_{V}^{2} \leq c\left(1+\|\vartheta\|_{V_{0}}^{2}\right)\left\|\chi_{t}\right\|_{H}^{2}
$$

Thus, on account of (3.7) and (3.9), a further use of the uniform Gronwall Lemma gives

$$
\begin{aligned}
\left\|\chi_{t}(t+r)\right\|_{H}^{2} & \leq c\left(E(0) e^{-\varepsilon t}+c_{f}\right)\left[r^{-1}+c\left(E(0) e^{-\varepsilon t}+c_{f}\right)\left(1+r^{-1}\right)\right] \\
& \leq c\left(E(0) e^{-\varepsilon t}+r^{-1}+c_{f}\right)^{2} \quad \text { for all } t \geq r
\end{aligned}
$$

and integrating (3.14) over $(t, t+r)$ for $t \geq 2 r$, thanks to (3.15) we have

$$
\int_{t}^{t+r}\left\|\chi_{t}(s)\right\|_{V}^{2} \mathrm{~d} s \leq c\left(E(0) e^{-\varepsilon t}+r^{-1}+c_{f}\right)^{2} \quad \text { for all } t \geq 2 r .
$$

Fourth estimate. A comparison argument in relation (2.12), together with (3.7), (3.10), (3.16), and well-known elliptic regularity results, immediately yields

$$
\int_{t}^{t+r}\|\vartheta(s)\|_{D(B)}^{2} \mathrm{~d} s \leq c\left(E(0) e^{-\varepsilon t}+r^{-1}+c_{f}\right)^{2}, \quad \text { for all } t \geq 2 r .
$$

Fifth estimate. Testing (2.13) by $A \chi$, using the monotonicity of $\beta$, and relying on estimates (3.6) and (3.15), we easily get

$$
\|\chi(t)\|_{D(A)}^{2} \leq c\left(E(0) e^{-\varepsilon t}+r^{-1}+c_{f}\right)^{2} \text { for all } t \geq 2 r .
$$


Next, a comparison in (2.13) immediately permits us to obtain

$$
\left\|\beta^{0}(\chi(t))\right\|_{H}^{2} \leq c\left(E(0) e^{-\varepsilon t}+r^{-1}+c_{f}\right)^{2} \text { for all } t \geq 2 r .
$$

Let us point out that this comparison argument, which is rigorous as far as $\beta$ is a smooth function (so that $\beta \equiv \beta^{0}$ ), is just formal when $\beta$ is multivalued. This procedure is fully justified, e.g., in [43, Proof of Theorem 2.16], where it is also explained why the minimal section $\beta^{0}$ appears.

Sixth estimate. Let us observe that $\vartheta$ solves

$$
\vartheta_{t}+B \vartheta=g \quad \text { a.e. in } \Omega \times(0,+\infty),
$$

where $g:=f-\lambda^{\prime}(\chi) \chi_{t}$. Recalling (H6) and using (3.7), (3.16), (3.18), and (2.4) it is not difficult to get

$$
\begin{aligned}
\int_{t}^{t+r}\|g(s)\|_{V}^{2} \mathrm{~d} s & \leq c_{f}+c \int_{t}^{t+r}\left\|\chi_{t}(s)\right\|_{V}^{2}\left(1+\|\chi(s)\|_{V}\|\chi(s)\|_{D(A)}\right) \mathrm{d} s \\
& \leq\left(E(0) e^{-\varepsilon t}+r^{-1}+c_{f}\right)^{\frac{7}{2}} \quad \text { for all } t \geq 2 r .
\end{aligned}
$$

Thus, $g(\cdot)$ is in $V$. However, in general it does not belong to $V_{0}=D\left(B^{1 / 2}\right)$. Nevertheless, if $\sigma \in\left(0, \frac{1}{4}\right), V \subset D\left(B^{\sigma}\right)$ with continuous embedding. Thus, testing (2.12) by $B^{2 \sigma} \vartheta$ and using (H6), (3.17), and the uniform Gronwall lemma, we infer

$$
\|\vartheta(t)\|_{D\left(B^{(1+2 \sigma) / 2}\right)}^{2} \leq c\left(E(0) e^{-\varepsilon t}+r^{-1}+c_{f}\right)^{\frac{7}{2}} \text { for all } t \geq 3 r .
$$

Let us point out that, as $\sigma \in\left(0, \frac{1}{4}\right)$, it is

$$
D\left(B^{(1+2 \sigma) / 2}\right) \subset V \quad \text { with compact embedding. }
$$

However, in three dimensions of space $D\left(B^{(1+2 \sigma) / 2}\right)$ is not included into $C(\bar{\Omega})$. Thus, a further bound is required.

Seventh estimate. Here we estimate $\|\vartheta(t)\|_{C(\bar{\Omega})}$ for sufficiently large $t$. Let us recall (3.20) and notice that, by (3.7) and (2.4) it is also

$$
\int_{t}^{t+r}\|g(s)\|_{H}^{2} \mathrm{~d} s \leq E(0) e^{-\varepsilon t}+c_{f} \text { for all } t \geq 0 .
$$

Then, let us take, just for the sake of simplicity, $r=1$ and consider $t \geq 3$. Let $N=N(t) \in \mathbb{N}$ such that $t \in(N, N+1]$ and decompose $\vartheta=\vartheta^{1}+\vartheta^{2}$, where

$$
\begin{array}{ll}
\vartheta_{t}^{1}+B \vartheta^{1}=g^{1}, & \vartheta^{1}(0)=\vartheta_{0} \\
\vartheta_{t}^{2}+B \vartheta^{2}=g^{2}, & \vartheta^{2}(0)=0 .
\end{array}
$$


Here

$$
g^{1}:=g \zeta([0, N-1]), \quad g^{2}:=g \zeta((N-1, N+1)),
$$

$\zeta(J)$ denoting the characteristic function of the interval $J$. For the solution of the equation (3.24) we have the representation formula

$$
\vartheta^{1}(t)=e^{B t} \vartheta_{0}+\int_{0}^{N-1} e^{B(t-s)} g^{1}(s) \mathrm{d} s .
$$

Let $X^{\alpha}=D\left(B^{\alpha}\right)$ endowed with the graph norm $\|\cdot\|_{\alpha}$. Then $X^{\alpha} \subset C(\bar{\Omega})$ for $\alpha>\frac{3}{4}$ and the following estimate holds:

$$
\left\|B^{\alpha} e^{B t} u\right\|_{H} \leq \kappa t^{-\alpha} e^{-\mu t}\|u\|_{H}
$$

for some $\kappa>0$ and some $\mu>0$. It is now sufficient to estimate $\left\|B^{\alpha} \vartheta(t)\right\|_{H}$, the $H$-norm being estimated by (2.3) and (3.9). We have

$$
B^{\alpha} \vartheta^{1}(t)=B^{\alpha} e^{B t} \vartheta_{0}+\int_{0}^{N-1} B^{\alpha} e^{B(t-s)} g^{1}(s) \mathrm{d} s .
$$

The first term decays exponentially by (3.26). Concerning the second, on account of (3.23) and (3.26), we infer

$$
\begin{aligned}
\int_{0}^{N-1}\left\|B^{\alpha} e^{B(t-s)} g^{1}(s)\right\|_{H} \mathrm{~d} s & \leq c \sum_{n=0}^{N-2} \int_{n}^{n+1} e^{-\mu(t-s)}\left\|g^{1}(s)\right\|_{H} \mathrm{~d} s \\
& \leq c \sum_{n=0}^{N-2} e^{-\mu(N-n-1)} \int_{n}^{n+1}\left\|g^{1}(s)\right\|_{H} \mathrm{~d} s \\
& \leq c \sum_{n=0}^{N-2} e^{-\mu(N-n-1)}\left(E(0) e^{-\varepsilon n}+c_{f}\right)^{\frac{1}{2}} \\
& \leq c E(0) e^{-\mu(N-1)} \frac{e^{\left(\mu-\frac{\varepsilon}{2}\right)(N-1)}-1}{e^{\mu-\varepsilon}-1}+c_{f} \\
& \leq c E(0) e^{-m t}+c_{f}
\end{aligned}
$$

where we have set $m:=\min \left\{\mu, \frac{\varepsilon}{2}\right\}$ and the last constants $c, c_{f}$ depend, of course, also on $\varepsilon, \mu$. Therefore, for any $t \geq 3$ we deduce

$$
\left\|\vartheta^{1}(t)\right\|_{X^{\alpha}}^{2} \leq c E(0) e^{-m t}+c_{f}
$$

Consider now equation (3.25) and observe that (3.20) implies

$$
\left\|g^{2}\right\|_{L^{2}\left((N-1, N+1) ; L^{6}(\Omega)\right)} \leq c\left(E(0) e^{-\varepsilon t}+c_{f}\right)^{\frac{7}{4}} .
$$


Thus we can apply the maximal regularity result [29] to obtain

$$
\begin{aligned}
\left\|\vartheta^{2}\right\|_{L^{2}\left((0, N+1) ; W^{2,6}(\Omega)\right)}+\left\|\vartheta_{t}^{2}\right\|_{L^{2}\left((0, N+1) ; L^{6}(\Omega)\right)} & \leq c\left\|g^{2}\right\|_{L^{2}\left((N-1, N+1) ; L^{6}(\Omega)\right)} \\
& \leq c\left(E(0) e^{-\varepsilon t}+c_{f}\right)^{\frac{7}{4}} .
\end{aligned}
$$

Then, the embedding theorem [6, Chapter III, Theorem 4.10.2] entails

$$
\left\|\vartheta^{2}\right\|_{B U C\left([0, N+1] ;\left(L^{6}(\Omega), W^{2,6}(\Omega)\right)_{1 / 2,2}\right.} \leq c\left(E(0) e^{-\varepsilon t}+c_{f}\right)^{\frac{7}{4}} .
$$

We recall that the interpolation space $\left(L^{6}(\Omega), W^{2,6}(\Omega)\right)_{1 / 2,2}=\mathcal{B}_{6,2}^{1}(\Omega)$ is continuously embedded into $C(\bar{\Omega})$. This gives a bound for $\left\|\vartheta^{2}(t)\right\|_{C(\bar{\Omega})}$ which is still independent of $N$. Taking $\alpha>\frac{3}{4}$ and summing up (3.28) and (3.29), we finally deduce

$$
\|\vartheta(t)\|_{C(\bar{\Omega})} \leq M_{1} E(0)^{\frac{1}{2}} e^{-\frac{m t}{2}}+M_{2} E(0)^{\frac{7}{4}} e^{-\frac{7 \varepsilon t}{4}}+M_{f}
$$

for some constant $M_{1}, M_{2}, M_{f}>0$, among which only $M_{f}$ depends on $f$, and for any $t \geq 3$. This completes the proof of Theorem 2.1 in the case of $r=1$ (cf. (2.16)). The modifications required to deal with a general $r>0$ will be outlined below.

3.2. Proof of the separation property (Theorem 2.3). Recalling (2.4), we set

$$
R_{0}=L M_{f}
$$

Then, if $R>R_{0}$, i.e., the compatibility condition holds, on account of (3.30) we can choose $T_{0} \geq 3$ such that

$$
L M_{1} E(0)^{\frac{1}{2}} e^{-\frac{m T_{0}}{2}}+L M_{2} E(0)^{\frac{7}{4}} e^{-\frac{7 \varepsilon T_{0}}{4}}<R-R_{0} .
$$

Therefore the function

$$
u \mapsto \phi(u):=L \max \left\{|\vartheta(x, t)|, x \in \bar{\Omega}, t \geq T_{0}\right\}-\partial W(u)
$$

is strictly negative in a left neighborhood of $u=1$. We remark once more that $\phi$ is actually a multifunction. Nevertheless, we shall still reason as if $\phi$ were smooth, since considering the general (multivalued) case gives notation problems only, but no further technical complications.

Consequently, if $1 \in D(\beta)$, then we can compare $\chi$ with the solution $\bar{u}$ to the Cauchy problem

$$
\left\{\begin{aligned}
\bar{u}^{\prime} & =\phi(\bar{u}) \\
\bar{u}\left(T_{0}\right) & =1,
\end{aligned}\right.
$$

and the comparison principle immediately entails, for any $t \geq T_{0}$,

$$
\chi(x, t) \leq \bar{u}(t) \quad \forall x \in \bar{\Omega} .
$$


If $(\mathrm{H} 7 \mathrm{~s})$ holds, then (3.34) can be modified by taking instead

$$
\left\{\begin{aligned}
\bar{u}^{\prime} & =\phi(\bar{u}) \\
\bar{u}\left(T_{0}\right) & =\max \left\{|\chi(x, t)|, x \in \bar{\Omega}, t \geq T_{0}\right\},
\end{aligned}\right.
$$

where the right hand side of the last equation is a uniformly bounded quantity in view of estimate (3.18). In both cases, thanks to the properties of $\phi$, it is clear that the upper bound in (S) holds by choosing any $T>T_{0}$ and

$$
\delta=\delta^{+}:=1-(\max \{u: \phi(u)=0\} \vee \bar{u}(T)) .
$$

Next, the procedure can be repeated (with obvious adjustments) in order to get the lower bound in $(\mathrm{S})$ with respect to a possibly different number $\delta^{-}>0$. Coupling the two bounds leads exactly to $(\mathrm{S})$ with $\delta:=\min \left\{\delta^{+}, \delta^{-}\right\}$. This ends the proof.

Proof of Corollary 2.5. We proceed along the same lines as before. However, we have to refine the Seventh Estimate in the proof of Theorem 2.1 by making explicit the dependence on $r>0$ (and not just take $r=1$ ). We do not give the technical details. The main difference is that now one has to take a generic $t \geq 3 r$ and choose $N \in \mathbb{N}$ such that $t \in(N r,(N+1) r]$. Then, one replaces the domains of integration accordingly and repeats all the subsequent procedure, taking care of the dependence on $r$ of the various constants. The evident conclusion is that the constant $R_{0}$ defined in (3.31) is itself depending on $r$ as well (and, of course, it blows up as $r \searrow 0$ ). Nevertheless, since we now have the strong coercivity $(\mathrm{H} 7 \mathrm{~s})$, the function $\phi$ defined in (3.33) will be strictly negative in a left neighborhood of $u=1$ whatever is the value taken for $T_{0}>0$ (namely, we do not need to make the choice (3.32)). At this point, we conclude as before by a comparison argument with respect to the system (3.36).

Proof of Corollary 2.6. The assertion follows by repeating once more the argument from (3.33) and noting that $\vartheta(t) \rightarrow 0$ as $t \rightarrow+\infty$ in the space $C(\bar{\Omega})$, which is proved in the next Lemma 3.1.

3.3. Proof of Theorem 2.7. Recalling (3.1) and using (H8) together with (3.6), we deduce

$$
\|\vartheta\|_{L^{2}\left(0,+\infty ; V_{0}\right)}+\left\|\chi_{t}\right\|_{L^{2}(0,+\infty ; H)} \leq c_{f} .
$$

Then, thanks to (2.16) and (3.38), from equation (2.12) we infer

$$
\vartheta_{t}, B \vartheta \in L^{2}((1,+\infty) ; H) .
$$

Thus, using the uniform bound (3.21) and the compact immersion (3.22), it is not difficult to deduce that

$$
\vartheta(t) \rightarrow 0 \quad \text { in } V \quad \text { as } t \rightarrow+\infty \text {. }
$$


Furthermore, on account of (3.28) and (3.29) it is

$$
\vartheta \in C\left([3,+\infty) ; X^{\alpha}+\mathcal{B}_{6,2}^{1}(\Omega)\right) .
$$

Since both the spaces $X^{\alpha}$ (for $\alpha>\frac{3}{4}$ ) and $\mathcal{B}_{6,2}^{1}(\Omega)$ are compactly embedded into $C(\bar{\Omega})$ we readily obtain

$$
\vartheta(t) \rightarrow 0 \quad \text { in } C(\bar{\Omega}) \quad \text { as } t \rightarrow+\infty
$$

On account of the above considerations and keeping (3.18) in mind, one easily realizes that the following lemma holds.

Lemma 3.1. Let the hypotheses (H1)-(H6) and (H8) be satisfied. Then

$$
\vartheta(t) \rightarrow 0 \quad \text { in } V_{0} \cap C(\bar{\Omega}) \quad \text { as } t \rightarrow+\infty \text {. }
$$

Moreover, any sequence of times $t_{n} \rightarrow+\infty$ contains a subsequence (not relabeled) such that

$$
\chi\left(t_{n}\right) \rightarrow \chi_{\infty} \quad \text { strongly in } V \cap C(\bar{\Omega}),
$$

where $\chi_{\infty}$ is a solution to (2.18).

Hence, to conclude the proof we have to show, thanks to the analyticity of $W$, the whole convergence of $\chi(t)$ to $\chi_{\infty}$ (not just along subsequences). Recall that we assume $f \equiv 0$ just for the sake of simplicity (cf. Remark 2.8, however). We proceed in the same way as in [2], so we just outline the argument.

We first observe that the separation property $(\mathrm{S})$ holds with $R_{0}=0$, due to Corollary 2.6, for some $T>0$ and some $\delta>0$. On the other hand, a comparison argument entails

$$
\max _{x \in \Omega}\left|\chi_{\infty}(x)\right|<1
$$

More precisely, since the set of all stationary solutions is compact in $C(\bar{\Omega})$, then there exists $\delta_{0}>0$ such that

$$
\max _{x \in \Omega}\left|\chi_{\infty}(x)\right| \leq 1-\delta_{0}
$$

for any stationary solution $\chi_{\infty}$, and $\left|W^{\prime}(y)\right|>0$ for $|y|>1-\delta_{0}$. We can thus modify $W^{\prime}$ outside of $\left(-1+\frac{\delta_{1}}{2}, 1-\frac{\delta_{1}}{2}\right)$, with $\delta_{1}:=\min \left\{\delta, \delta_{0}\right\}$, in such a way that

$$
\begin{aligned}
\mathcal{F}(y) & =W^{\prime}(y) \quad \forall y \in\left[-1+\delta_{1}, 1-\delta_{1}\right], \\
|\mathcal{F}(y)| & \leq c,\left|\mathcal{F}^{\prime}(y)\right| \leq c \quad \forall y \in \mathbb{R} .
\end{aligned}
$$

To conclude the proof it suffices to show that there exists $T_{1}>T$ such that $\chi_{t} \in L^{1}\left(T_{1},+\infty ; H\right)$. In fact, consider first the energy inequality (3.1). It is clear that $E$ defined by (3.4) is non-increasing in $(0,+\infty)$. Therefore, on account 
of (3.40), we have that $E(t)$ converges, as $t$ goes to $+\infty$, to $I_{\infty}:=I\left(\chi_{\infty}\right)$, where $I$ is the functional defined by

$$
I(v):=\frac{1}{2}\|\nabla v\|_{H}^{2}+\int_{\Omega} W(v) \mathrm{d} x .
$$

Integrate now the energy inequality (3.1) with respect to time from $t>0$ to $+\infty$. This gives

$$
\int_{t}^{+\infty}\left(\frac{\|\vartheta(s)\|_{V_{0}}^{2}}{2}+\left\|\chi_{t}(s)\right\|_{H}^{2}\right) \mathrm{d} s \leq \frac{\|\vartheta(t)\|_{H}^{2}}{2}+I(\chi(t))-I_{\infty} .
$$

Since $\chi_{\infty}$ is smooth enough, we can estimate $I(\chi(t))-I_{\infty}$ by a modified version of the Łojasiewicz-Simon inequality. Indeed, following [2, Proposition 4.2], we have that, for any $P>0$, there exist $\rho \in\left(0, \frac{1}{2}\right)$ and $\delta_{2}=\delta_{2}(P) \in\left(0, \delta_{1}\right), c_{P}>0$ such that

$$
\|A(\bar{\chi})+\partial W(\bar{\chi})\|_{V^{\prime}} \geq c_{P}\left|I(\bar{\chi})-I\left(\chi_{\infty}\right)\right|^{1-\rho},
$$

for all $\bar{\chi} \in V$ such that $\left\|\bar{\chi}-\chi_{\infty}\right\|_{H}<\delta_{2}$ and $\left|I(\bar{\chi})-I\left(\chi_{\infty}\right)\right|<P$.

Thus, from (3.43) we infer (cf. also (2.3))

$$
\int_{t}^{+\infty}\left(\left\|\chi_{t}(s)\right\|_{H}^{2}+\|\vartheta(s)\|_{V_{0}}^{2}\right) \mathrm{d} s \leq c\left(\left\|\chi_{t}(t)\right\|_{H}^{2}+\|\vartheta(t)\|_{V_{0}}^{2}\right)^{\frac{1}{2-2 \rho}},
$$

for any $t \in \mathcal{M}:=\left\{t>0:\left\|\chi(t)-\chi_{\infty}\right\|_{H}<\delta_{2}\right\}$. This implies (see [19, Lemma 7.1], cf. also [2, Lemma 5.1]) $\left\|\chi_{t}(\cdot)\right\|_{H} \in L^{1}(\mathcal{M})$, which combined with (3.41) and a simple contradiction argument yields $\left(T_{1},+\infty\right) \subset \mathcal{M}$ for some $T_{1}>T$ large enough. Hence, we obtain $\left\|\chi_{t}(\cdot)\right\|_{H} \in L^{1}\left(T_{1},+\infty\right)$ which entails

$$
\chi(t) \rightarrow \chi_{\infty} \quad \text { strongly in } H, \quad \text { as } t \rightarrow+\infty .
$$

Thus, on account of (3.41), the second assertion of Theorem 2.7 follows.

To prove the decay rate estimate, we adapt an argument used, for instance, in [28]. On account of (3.1), we have

$$
\frac{\mathrm{d}}{\mathrm{d} t} E(t) \leq-c\left(\|\vartheta(t)\|_{H}^{2}+\left\|\chi_{t}(t)\right\|_{H}^{2}\right) \quad \forall t \geq 0
$$

Using again (3.44), recalling (2.4), and taking advantage of equation (2.13) and (3.39), we have, for $t>0$ large enough,

$$
\begin{aligned}
E(t)-I_{\infty} & \leq c\left(\|\vartheta(t)\|_{H}^{2}+\|A \chi(t)+\partial W(\chi(t))\|_{H}^{\frac{1}{1-\rho}}\right) \\
& \leq c\left(\|\vartheta(t)\|_{H}+\left\|\lambda^{\prime}(\chi(t)) \vartheta(t)-\chi_{t}(t)\right\|_{H}\right)^{\frac{1}{1-\rho}} \\
& \leq c\left(\|\vartheta(t)\|_{H}+\left\|\chi_{t}(t)\right\|_{H}\right)^{\frac{1}{1-\rho}} .
\end{aligned}
$$


Thus, we deduce

$$
\left(E(t)-I_{\infty}\right)^{2(1-\rho)} \leq c\left(\|\vartheta(t)\|_{H}^{2}+\left\|\chi_{t}(t)\right\|_{H}^{2}\right) .
$$

Inequalities (3.45) and (3.46) then yield $\frac{\mathrm{d}}{\mathrm{d} t}\left(E(t)-I_{\infty}\right)+c\left(E(t)-I_{\infty}\right)^{2(1-\rho)} \leq 0$, so that

$$
E(t)-I_{\infty} \leq c(1+t)^{-\frac{1}{1-2 \rho}}
$$

On the other hand, we have $-\frac{\mathrm{d}}{\mathrm{d} t}\left(E(t)-I_{\infty}\right)^{\rho}=-\rho \frac{\mathrm{d}}{\mathrm{d} t} E(t)\left(E(t)-I_{\infty}\right)^{\rho-1}$. Thus, thanks to (3.45) and (3.46), we infer

$$
-\frac{\mathrm{d}}{\mathrm{d} t}\left(E(t)-I_{\infty}\right)^{\rho} \geq c\left(\|\vartheta(t)\|_{H}+\left\|\chi_{t}(t)\right\|_{H}\right)
$$

This entails $\frac{1}{c}\left(E(t)-I_{\infty}\right)^{\rho} \geq \int_{t}^{+\infty}\left(\|\vartheta(s)\|_{H}+\left\|\chi_{t}(s)\right\|_{H}\right) \mathrm{d} s$, and (2.20) follows from (3.47).

Acknowledgement. This work was partially supported by the Italian MIUR PRIN Research Projects Modellizzazione Matematica ed Analisi dei Problemi a Frontiera Libera and Aspetti Teorici e Applicativi di Equazioni a Derivate Parziali, and by the Italian MIUR FIRB Research Project Analisi di Equazioni a Derivate Parziali, Lineari e Non Lineari: Aspetti Metodologici, Modellistica, Applicazioni. The work of Hana Petzeltová was supported by the Grant A1019302 of GA AV ČR. The work of Giulio Schimperna was partially supported by the HYKE Research Training Network.

\section{References}

[1] Aizicovici, S. and Feireisl, E., Long-time stabilization of solutions to a phasefield model with memory. J. Evol. Equ. 1 (2001), 69 - 84.

[2] Aizicovici, S., Feireisl, E. and Issard-Roch, F., Long time convergence of solutions to a phase-field system. Math. Methods Appl. Sci. 24 (2001), $277-287$.

[3] Aizicovici, S. and Petzeltová, H., Compactness and long-time stabilization of solutions to phase-field models. In: Analysis and optimization of differential systems (Constanta, 2002). Boston (MA): Kluwer Acad. Publ. 2003, pp. $1-12$.

[4] Aizicovici, S. and Petzeltová, H., Asymptotic behavior of solutions of a conserved phase-field system with memory. J. Integral Equations Appl. 15 (2003), $217-240$.

[5] Aizicovici, S. and Petzeltová, H., Convergence of solutions of phase-field systems with a nonconstant latent heat. Dynam. Systems Appl. 14 (2005), $163-173$. 
[6] Amann, H., Linear and Quasilinear Parabolic Problems. Basel: Birkhäuser 1995.

[7] Aulbach, B., Continuous and discrete dynamics near manifolds of equilibria. Berlin: Springer 1984.

[8] Barbu, V., Nonlinear Semigroups and Differential Equations in Banach Spaces. Leyden: Noordhoff 1976.

[9] Bates, P. W. and Zheng, S., Inertial manifolds and inertial sets for phase-field equations. J. Dynamics Differential Equations 4 (1992), 375 - 397.

[10] Brezis, H., Opérateurs Maximaux Monotones et Sémi-groupes de Contractions dans les Espaces de Hilbert. Amsterdam: North-Holland Math. Studies 5, 1973.

[11] Brochet, D., Chen, X. and Hilhorst, D., Finite dimensional exponential attractor for the phase-field model. Appl. Anal. 49 (1993), 197 - 212.

[12] Brochet, D. and Hilhorst, D., Universal attractor and inertial sets for the phase-field model. Appl. Math. Lett. 4 (1991), 59 - 62.

[13] Brokate, M. and Sprekels, J., Hysteresis and Phase Transitions. New York: Springer 1996.

[14] Caginalp, G., An analysis of a phase field model of a free boundary. Arch. Rational Mech. Anal. 92 (1986), 205 - 245.

[15] Chill, R., On the Łojasiewicz-Simon gradient inequality. J. Funct. Anal. 201 (2003), $572-601$.

[16] Chill, R. and Jendoubi, M. A., Convergence to steady states in asymptotically autonomous semilinear evolution equations. Nonlinear Anal. 53 (2003), $1017-1039$.

[17] Damlamian, A., Kenmochi, N. and Sato, N., Subdifferential operator approach to a class of nonlinear systems for Stefan problems with phase relaxation. Nonlinear Anal. 23 (1994), 115 - 142.

[18] Elliott, C. M. and Zheng, S., Global existence and stability of solutions to the phase field equations. In: Free boundary value problems (Oberwolfach, 1989). Internat. Ser. Numer. Math. 95. Basel: Birkhäuser 1990, pp. 46 - 58.

[19] Feireisl, E. and Simondon, F., Convergence for semilinear degenerate parabolic equations in several space dimensions. J. Dynam. Differential Equations 12 (2000), $647-673$.

[20] Feireisl, E., Issard-Roch, F. and Petzeltová, H., Long-time behaviour and convergence towards equilibria for a conserved phase field model. Discrete Contin. Dyn. Syst. 10 (2004), $239-252$.

[21] Feireisl, E., Issard-Roch, F. and Petzeltová, H., A non-smooth version of the Lojasiewicz-Simon theorem with applications to non-local phase-field systems. J. Differential Equations 199 (2004), 1 - 21.

[22] Feireisl, E. and Schimperna, G., Large time behavior of solutions to PenroseFife change models. Math. Methods Appl. Sci. 28 (2005), 2117 - 2132. 
[23] Giorgi, C., Grasselli, M. and Pata, V., Uniform attractors for a phase-field model with memory and quadratic nonlinearity. Indiana Univ. Math. J. 48 (1999), $1395-1445$.

[24] Grasselli, M. and Pata, V., Asymptotic behavior of a parabolic-hyperbolic system. Commun. Pure Appl. Anal. 3 (2004), 849 - 881.

[25] Grasselli, M., Petzeltová, H. and Schimperna, G., Convergence to stationary solutions for a parabolic-hyperbolic phase-field system. Commun. Pure Appl. Anal. (to appear).

[26] Haraux, A., Systèmes Dynamiques Dissipatifs et Applications. Paris: Masson 1991.

[27] Haraux, A. and Jendoubi, M. A., Convergence of bounded weak solutions of the wave equation with dissipation and analytic nonlinearity. Calc. Var. Partial Differential Equations 9 (1999), 95 - 124.

[28] Haraux, A., Jendoubi, M. A. and Kavian, O., Rate of decay to equilibrium in some semilinear parabolic equations. J. Evol. Equ. 3 (2003), 463 - 484.

[29] Hieber, M. and Prüss, J., Heat kernels and maximal $L^{p}-L^{q}$ estimates for parabolic evolution equations. Commun. in Partial Differential Equations 22 (1997), $1647-1669$.

[30] Horn, W., Sprekels, J. and Zheng, S., Global existence of smooth solutions to the Penrose-Fife model for Ising ferromagnets. Adv. Math. Sci. Appl. 6 (1996), $227-241$.

[31] Huang, S.-Z. and Takáč, P., Convergence in gradient-like systems which are asymptotically autonomous and analytic. Nonlinear Anal. 46 (2001), 675 - 698.

[32] Jendoubi, M. A., A simple unified approach to some convergence theorems of L. Simon. J. Funct. Anal. 153 (1998), $187-202$.

[33] Jendoubi, M. A., Convergence of global and bounded solutions of the wave equation with linear dissipation and analytic nonlinearity. J. Differential Equations 144 (1998), 302 - 312.

[34] Jiménez-Casas, A. and Rodríguez-Bernal, A., Asymptotic behaviour for a phase field model in higher order Sobolev spaces. Rev. Mat. Complut. 15 (2002), $213-248$.

[35] Kalantarov, V. K., On the minimal global attractor of a system of phase field equations (in Russian). Zap. Nauchn. Sem. Leningrad. Otdel. Mat. Inst. Steklov (LOMI) 188 (1991). Kraev. Zadachi Mat. Fiz. i Smezh. Voprosy Teor. Funktsii 22, 70 - 86, 186; translation in: J. Math. Sci. 70 (1994), 1767 - 1777.

[36] Laurençot, Ph., Long-time behaviour for a model of phase-field type. Proc. Roy. Soc. Edinburgh Sect. A 126 (1996), $167-185$.

[37] Łojasiewicz, S., Une propriété topologique des sous ensembles analytiques réels. In: Colloques internationaux du C.N.R.S. 117: Les équations aux dérivées partielles (Paris, 1962). Paris: Editions du C.N.R.S. 1963, pp. 87 - 89.

[38] Łojasiewicz, S., Ensembles semi-analytiques. Bures-sur-Yvette: Notes I.H.E.S. 1965. 
[39] McFadden, G. B., Phase-field models of solidification. Contemp. Math. 306 (2002), $107-145$.

[40] Miranville, A. and Zelik, S., Robust exponential attractors for Cahn-Hilliard type equations with singular potentials. Math. Methods Appl. Sci. 27 (2004), $545-582$.

[41] Poláčik, P. and Rybakowski, K. P., Nonconvergent bounded trajectories in semilinear heat equations. J. Differential Equations 124 (1996), 472 - 494.

[42] Poláčik, P. and Simondon, F., Nonconvergent bounded solutions of semilinear heat equations on arbitrary domains. J. Differential Equations 186 (2002), $586-610$.

[43] Rocca, E. and Schimperna, G., Universal attractor for some singular phase transition systems. Phys. D 192 (2004), 279 - 307.

[44] Rybka, P. and Hoffmann, K.-H., Convergence of solutions to Cahn-Hilliard equation. Comm. Partial Differential Equations 24 (1999), 1055 - 1077.

[45] Schimperna, G., Abstract approach to evolution equations of phase field type and applications. J. Differential Equations 164 (2000), 395 - 430.

[46] Simon, L., Asymptotics for a class of non-linear evolution equations, with applications to geometric problems. Ann. Math. 118 (1983), 525 - 571.

[47] Temam, R., Infinite-Dimensional Dynamical Systems in Mechanics and Physics. New York: Springer Verlag 1997.

[48] $\mathrm{Wu}, \mathrm{H}$. and Zheng, S., Convergence to equilibrium for the Cahn-Hilliard equation with dynamic boundary condition. J. Differential Equations 204 (2004), $511-531$.

[49] Zhang, Z., Asymptotic behavior of solutions to the phase-field equations with Neumann boundary conditions. Comm. Pure Appl. Anal. (to appear).

Received January 11, 2005 